\title{
Endometrial sarcoma. Case report and review of the literature
}

\author{
Spyroulis C*, Sumaira A and Sarhanis $\mathbf{P}$ \\ Truct Junior Registrar in Obstetrics and Gynaecology, England
}

Received: November 04, 2017; Published: November 13, 2017

*Corresponding author: Spyroulis C, Truct Junior Registrar in Obstetrics and Gynaecology at Northwick Park hospital, Consultant Gynaecology, Lead for Gynaecology Oncology at LNWH, England; Email: c.spyroulis@nhs.net

Abstract

Endometrial stromal sarcoma is a rare type of endometrial cancer that is mainly present in older women. There is no specific classification for this type of endometrial cancer and for this reason we use the FIGO classification that is used to stage endometrial type cancers. The main presenting complaint of women with endometrial stromal sarcoma is PV bleeding. Endometrial sarcoma cells are positive for both estrogen and progesterone receptors and have high levels of CD-10 and inhibin. We present a case of a 56 year old lady who came to the RAC due to PMB and scan findings suggesting of endometrial fibroid. Patient undergone a hysteroscopy which shown a large polypoid lesion in the posterior uterine wall and a large fibroid tissue lesion in the endometrium, suggesting of endometrial sarcoma. Patient had MRI and discussed in MDT, as per Trust policy and she had a TAH+BSO. The histology confirmed the diagnosis of low gradeendometrial sarcoma.

\section{Introduction}

Endometrial uterine sarcoma is a very rare tumour of the uterine cavity. It accounts for $0.2 \%$ of the total uterine malignancies. The annual incidence for this disease is 10-12/1.000.000 cases and the mean age is between 42-58 years. Despite being very rare, is an extremely indolent malignant tumour with local recurrences and distant metastases even 20 years the initial presentation of the disease:

\section{Cytogenetics}

Micci et al. [1] in 2006 identified a specific translocation T(7;17) (p15;q21 with involvement of two zinc finger genes juxtaposed with another zinc finger protein 1 and joint juxtaposed with another Zinc protein 1 was described in most of the ESS. Halbwed et al. [2] study shown a strong correlation between chromosomal deletion on $7 \mathrm{p}$ and tumor development and progression.

\section{Pathogenesis}

We don't know the exact pathogenesis of endometrial sarcoma, but PCOS, exposure to tamoxifen and unopposed estrogens, have been implicated in the pathogenesis of uterine sarcoma. WHO in 2003 classified endometrial sarcomas, into:

a) Endometrial stromal nodule,

b) Low-grade endometrial stromal sarcoma and

c) Undifferentiated endometrial or uterine sarcoma

\section{Diagnosis}

$90 \%$ of endometrial sarcomas will present with abnormal uterine bleeding and $70 \%$ will be associated with uterine enlargement, too. It is also possible to present with pelvic pain and dysmenorrhea. $25 \%$ of individuals will be asymptomatic. Tavasolli et al [3], study shows that in 30 to $50 \%$ of cases, by the time of diagnosis, endometrial sarcoma was already spread into neighbor organs. Ganjoei TA et al and Jin Y et al study, shown that, in the majority of cases, endometrial scrapping is useful in the diagnosis of the disease, because it involves both the myometrium and endometrium. Both studies shows that, if the disease is isolated in the myometrium, then endometrial scrapping won't be helpful. Additionally, due to the fact that, endometrial sarcoma has similarities with the normal endometrium, many times is impossible to put the definitive diagnosis based on the endometrial curettage, and we need to await the histologic diagnosis from the hysterectomy specimen.

\section{Radiology}

Ultrasound is not a reliable way to diagnose endometrial sarcoma, because of the similar picture with adenomyosis or uterine leiomyoma. MRI can be useful for the preoperative diagnosis, because it has the advantage to show possible metastases and gives more detailed information regarding the endometrial cavity. The presence of low-signal intensity within the area of myometrial 
invasion is suggestive of endometrial sarcoma. Additionally, continuous extension of the lesion into the adjacent structures along vessels, fallopian tubes, ligaments and ovaries is diagnostic of endometrial sarcoma.

\section{Immunohistochemistry}

CD10 is a cell surface neutral endopeptidase and Zhu XQ et al. (4), endometrial sarcoma cells express high levels of CD-10 and inhibin expression. Endometrial stromal cell tumors are positive for both estrogen and progesterone receptors

\section{Differential Diagnosis}

Endometrial sarcoma should be differentiated from neoplasms with arborizing vasculature, highly cellular leiomyoma, cellular endometrial polyp, low-grade mullarianadenosarcoma, and adenomyosis [5].

\section{Prognostic Factors}

Prognosis depends on the staging according to the FIGO classification. In the literature, there are several factor which have been associated with poor prognosis, like increased age, black race, DNA aneuploidy, proliferative activity, expression of hormone receptors, etc. Lai et al study, shown that, older patients ( $>50$ years), black race, advanced stage, lack of primary surgery, nodal metastasis, high mitotic count $>5 / 10$ high-power fields, CD10 negative or low expression or even lack of progesterone or estrogen receptors were independent factors for poor survival. However, we do know that, generally endometrial sarcomas have better survival rates than other sarcomas.

\section{Treatment}

The following are all potential treatment options for the management of stromal sarcomas:

\section{a. Surgery,}

b. Adjuvant therapy,

c. Hormone therapy and

d. Radiotherapy.

Surgery is the treatment of choice for endometrial stromal tumors. In case of undifferentiated endometrial sarcomas, patient should undergo debulking surgery for cytoreduction, in order to reduce the potential for metastasis. In case of endometrial stromal sarcomas, patient can undergo only hysterectomy with or without salpingoophorectomy. Because endometrial stromal tumors are hormonally sensitive, post-operative hormone replacement therapy, in case of TAH+BSO, is contraindicated. In case of young patients, we might think to preserve the ovaries, if the case is an endometrial stromal sarcoma type 1 , in order to avoid early menopause. Chan JK. Study, shown that $10 \%$ of those who underwent lymph node dissection had nodal metastases, and the recommendation was to undergo lympha denectomy for both prognostic, but also for therapeutic purposes. Additionally, patients with positive nodal metastasis had significant poorer prognosis than patients with negative nodes. The results of Chan et al study, supported also by several other studies and for this reason the recommendation is for lymphadenectomy, mainly for prognosis, since the therapeutic benefit, should be proved. Adjuvant therapy, should be considered in stage II-IV endometrial stromal sarcoma and involves hormone therapy with or without tumor dissected radiotherapy.

Hormone therapy is an option in case of endometrial stromal sarcoma, because these tumors have estrogen and progesterone receptors. Hormone therapy include: a) megestrol/medroxyprogesterone, gonadotrophin releasing hormone analogues and aromatase inhibitors, like letrazole and anastrozole. Spano JP et al. [6] and Alkasi et al. [7], are 2 case reports which shows 10 year free survival rate for the patients that received aromatase inhibitor for 10 years. Chu et al. [8], study compared the outcome for the patients who received adjuvant megestrol $160 \mathrm{mg} /$ day with those who didn't. The result was, that patients who received adjuvant megestrol, 75\% of them didn't have recurrence of the disease, whereas, patients who didn't have adjuvant megestrol, didn't have recurrence of the disease in $29 \%$. In case of recurrent disease, Maluf FC and Petal S suggest a dose of $2.5 \mathrm{mg}$ letrazole daily. Radiotherapy, is an option for the stage II-IV endometrial stromal sarcomas, but not for stage I.

\section{Recurrent Disease}

Recurrent disease is possible in 1/3-1/2 of cases and limited in the pelvis and lower genital tract. Distant metastasis can occur after years of disease. Chemotherapy is a mode of therapy for recurrent undifferentiated endometrial sarcoma, there is no strong evidence.

\section{Follow-Up And Survival Rates}

The 5 year survival rate for the FIGO type 1 is between $54-100 \%$. The relative 5 year survival rate for FIGO type 2 is $30 \%$, whereas for III-IV is only $10 \%$. Since the recurrence rate is very high with this type of tumor, it is essential to have a very thorough follow-up. It shall be once in 3months for the first year, and half yearly for next 4years. Thereafter, annual follow-up is recommended. The relapse free survival depends on the tumor stage, myometrial invasion, adjuvant therapy, and bilateral salpingo ophorectomy.

\section{Case Report}

We present a case of low grade endometrial sarcoma, which corresponds to endometrial stromal sarcoma according to the latest WHO classification (WHO 2003). A 56 year old was referred by her GP to the RAC of NPH due to postmenopausal bleeding PMB). Patient was P6, all normal deliveries and she noticed PMB for 3 days. She is taking amlodipine $5 \mathrm{mg}$ OD for hypertension, but otherwise fit and well, She didn't complain of any allergies and she was updated with the smear tests, which were all normal. She was seen on $15^{\text {th }}$ of November 2016, at the RAC of NPH by the Gynaecology Oncology lead. She had prior to the clinic (11 ${ }^{\text {th }}$ of November 2016) a scan which shown an endometrial thickness of $26 \mathrm{~mm}$ and a mass in the posterior wall of the uterus, which was typical of a fibroid. In the view of the PMB and the increased endometrial thickness in scan, a malignancy couldn't be excluded. She was examined by the gynae oncology lead and the findings were: abdomen soft, non-tender, no mass palpated. Speculum examination performed and cervix looked healthy and no obvious cause for PMB was seen. Pipelle attempted, 
but it was not successful because patient couldn't tolerate the examination. She was booked for an urgent hysteroscopy (2 week referral) with endometrial biopsy, as per Trust policy. On $28^{\text {th }}$ of November she had a hysteroscopy. Large polypoid lesion was seen in the lower segment of the uterine cavity. A large fibroid was seen in the endometrium and multiple pieces of this fibroid lesion taken and sent for biopsy via resectoscope. We were unable to completely remove the lesion from the lower uterine segment, since there was a high suspicion of being a cancer and we were afraid not to perforate the uterus and causing dissemination of the disease.

The plan was to send the patient for an MRI and to discuss the case in the MDT meeting. On $29^{\text {th }}$ of November, she had an MRI of the abdomen-pelvis. The scan shown a soft tissue which was arisen from the endometrial cavity which corresponds to an endometrial polyp and $4.4 \times 4.6 \mathrm{~cm}$ heterogenous mass from the posterior uterine wall, which is more suggestive of a fibroid or a malignant lesion arising from the myometrium including a sarcomatouslesion. On $29^{\text {th }}$ of November we had the results of histology which shown: low grade endometrial sarcoma. On $2^{\text {nd }}$ of December we discussed the case in the MDT meeting with the gynae oncology lead in Hammersmith hospital, which is a tertiary referral unit for North West London and the plan was for a total abdominal hysterectomy and bilateral salpingoophorectomy. The histology confirmed the diagnosis of a low grade endometrial stromal sarcoma. As per FIGO classification, the sarcoma was of stage $1 \mathrm{~B}$, since the tumor was confined to the myometrium. Microscopically, tumor cells express CD10, SMA, bcl-2 and CD99, but they were negative for desmin, h-caldesmon, melan-A, HMB45, S100 and CD34. We present this case, in order to point the complexity of the case, the rarity and of course, the importance of MDT meeting when you have high suspicion of cancer, without having the histological findings, yet.

\section{Discussion}

Endometrial sarcoma is a very rare malignancy of the endometrial cavity and is more frequent in older women. Sarcoma cells have very high hormonal affinity, both estrogenic and progesterone, they have high incidence of recurrence and for this reason follow-up is highly recommended even after the 5 year protocol and the treatment of choice is always total abdominal hysterectomy with bilateral salpingoophorectomy.

\section{References}

1. Micci F, Panagopoulos I, Bjerkehagen B, Heim S (2006) Consistent rearrangement of chromosomal band $6 \mathrm{p} 21$ with generation of fusion genes JAZF1/PHF1 and EPC1/PHF1 in endometrial stromal sarcoma. Cancer Res 66(1): 107-111.

2. Halbwed I, Ullmann R, Kremser ML, Man YG, Moud NI, et al. (2005) Chromosomal alterations in low-grade endometrial stromal sarcoma and undifferentiated endometrial sarcoma as detected by comparative genomic hybridization. GynecolOncol 97(2): 582-587.

3. Tavassoli FA, Devilee P (2003) WHO Classification of Pathology and genetics of tumours of the breast and female genital organs, In: IARC Press, Lyon, France, pp.233-236.

4. ZhuXQ Shi YF, ChengX,Zhao CL, WuYZ(2004) Detal Immunohistichemical markers in differential diagnosis of endometrial stromal sarcoma and cellular leomyoma. GynaecolOncol 92(1): 71-79.

5. Geetha Puliyath, Krishnan Nair (2012) Endometrial stromal sarcoma : A review of the literature. Indian Journal of Medical and PaediatricOncology 33(1): 1-6.

6. Spano JP, Soria JC, Kambouchner M, Piperno-Neuman S, Morin F, et al. (2003) Long-term survival of patients given hormonal therapy for metastatic endometrial stromal sarcoma. Med Oncol 20(1): 87-93.

7. Alkasi O, Meinhold-Heerlein I, Zaki R, Fasching P, Maass N, et al. (2009) Long-term disease-free survival after hormonal therapy of a patient with recurrent low grade endometrial stromal sarcoma: A case report. Arch Gynecol Obstet 279(1): 57-60.

8. Chu MC, Chu Mor G, Lim C, Zheng W, Prakash V, et al. (2003) Low-grade endometrial stromal sarcoma: Hormonal aspects. Gynaec Oncol 90(1): 170-176.

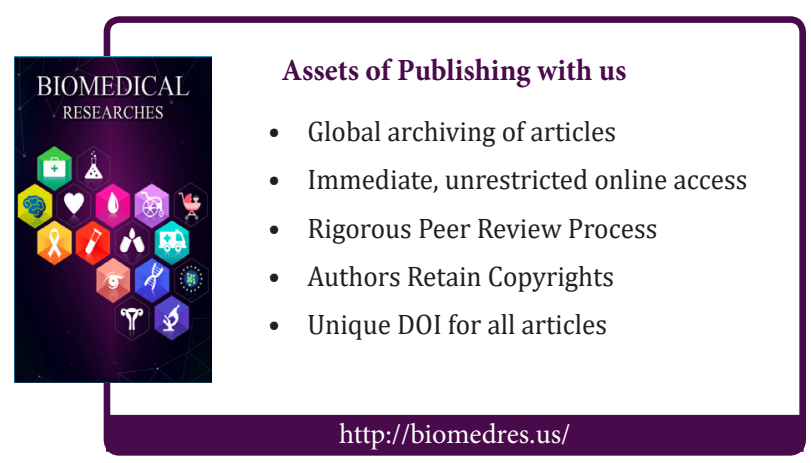

